\title{
CPAs And Electronic Survey Media
}

Marc A. Giullian, Ph.D., CPA (Email: mgiullian@montana.edu), Montana State University

Marcus D. Odom, Deloitte \& Touche Faculty Fellow, (Email: modom@ cba.siu.edu), Southern Illinois University - Carbondale Michael W. Totaro, (Email: mwtotaro@bplb.com), Broussard, Poche, Lewis \& Breaux, LLP, Louisiana

\begin{abstract}
Gathering and utilizing data about customers and other stakeholders has become vital to maintaining competitiveness in today's business world. With the ongoing expansion of the Internet, new methods for data collection are available. The success of utilizing new methods depends on the technological capabilities of the audience from whom data are sought. One group of business professionals perceived to possess above average technological skills is practicing Certified Public Accountants (CPAs). A study was designed to measure the response rates of CPAs to different survey administration methods. This study compares one traditional survey method (regular mail) with newer methods for collecting survey data (e-mail and WWW form).
\end{abstract}

\section{Introduction}

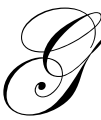
athering and utilizing data about customers and other stakeholders has become vital to maintaining competitiveness in today's business world. Traditionally, data collection has been conducted through mail questionnaires, telephone solicitations, customer response forms, and so on. With the ongoing expansion of the Internet, new methods for data collection are available. These new methods offer substantial benefits relative to traditional methods. However, much less is known about the efficacy of Internet-based data collection. One important factor that must be considered in using these newer forms of data collection is the technological capability of those from whom data is desired.

One group of business professionals perceived to possess above average technological skills is practicing Certified Public Accountants (CPAs). CPAs seek to stay up-to-date with new technology so they will be better able to help clients make use of these new technologies. Also, because many CPAs firms are providers of technologybased solutions to clients, it is no surprise that CPAs are viewed by many as having a strong technical background.

Results from a previous study showed that for a related population of accounting professionals (university accounting faculty) this perception, that accountants possess above average technological skills was not necessarily supported (Odom, et al 1998). In this study, regular mail surveys were more effective in generating responses than electronic surveys. This result was unexpected and begged the question of whether or not other groups of accounting professionals exhibit the same tendencies. CPAs were chosen because they share many characteristics with accounting faculty, (e.g. common body of knowledge, etc.), and also because the environment in which CPAs operate offers stronger monetary incentives for staying current with new technologies. This study compares one traditional survey method (regular mail) with newer methods (electronic communication media) for collecting survey data.

\section{Survey Methods}

\subsection{E-Mail}

Survey research via email is a relatively new alternative to traditional, mailed surveys. One similarity between these methods is that individuals have a unique address for both email and regular mail and can therefore receive personalized messages via either medium. Additionally, messages can be sent around the world and are delivered by a mechanism other than the sender. Finally, the communication process is essentially the same in that one person composes a message, sends it and often anticipates a response.

Readers with comments or questions are encouraged to contact the authors via email. 
One of the differences between regular mail and email is delivery speed. Blattberg and Glazer (1993) suggest that because of the speed at which email messages travel, communication processes that tended to be one-way now have the potential to be transformed into a two-way process. Feedback is sufficiently timely to be meaningful. This phenomenon is evident in "chat rooms" and at times when two people are use email simultaneously. Speed of delivery also enables the sender of email to know much sooner if the message has been sent to an undeliverable address. Another difference is that email is less costly than regular mail, especially in instances where a high volume of mail is sent. A difference of particular interest to researchers is that when a message is sent via email as opposed to regular mail, there is a higher likelihood that it will be read by the intended recipient. This is because the message is less likely to be screened by another person (Dyson, 1993). Thus, email may provide greater assurance that researchers are receiving data from the desired population. A further difference is that the population currently able to utilize email is much smaller and more homogeneous than those who receive regular mail. Those using email are more likely to have a college education and earn an above average income. Thus, the researcher must ensure the population most appropriate for the research question has access to this technology. An interesting behavioral difference is that respondents to email surveys tend to be more candid and frank than those responding to regular mail surveys (Kiesler \& Sproull, 1986). Researchers suggest that the cause of this behavioral difference is the reduced social context involved in communicating via email.

\subsection{WWW}

Surveys utilizing the WWW are quite different than regular mail surveys. An important difference is that the respondent must go and find the survey rather than having it come to him, as with a mail survey. Surveys are posted to a web site and anyone visiting the web site can complete the survey. This characteristic of WWW surveys results in the researcher having much less control over who responds. This is a potential concern because of selfselection bias. The researcher has no objective method of determining why those who responded decided to do so. One possible way to alleviate this potential problem may be to selectively request individuals to respond. This request will require the use of some form of solicitation such as regular mail or email.

A major benefit of using the WWW to gather data is that software is available to have responses input immediately into a predesigned database. Survey data does not need to be converted to an electronic form by the researcher. This benefit eliminates one of the time-consuming elements of survey research. This may reduce the overall cost of administering a survey by as much as 30 percent (Ferrerra and Nolan, 1974). The cost of this software is small, relative to the cost of manually inputting the data. ${ }^{1}$ Thus, the WWW mode of survey data gathering has the potential to significantly reduce one of the significant costs of survey research. Another difference is the WWW, while growing in size and popularity by astounding proportions, is available to a limited population--those who have access to the necessary hardware and software. This group is likely to have a disproportionate number who are educated and belong to an upper socio-economic class. However, as long as the desired population has access to the required technology, using the WWW is an attractive option.

\section{Expectations}

The a priori expectation regarding overall response rates was that they would be somewhat higher than 5 percent and probably no higher than 30 percent, consistent with prior empirical studies (Alreck \& Settle, 1985). The expected response rate for the regular mail surveys was also anticipated to be between 5 and 30 percent. We found very few prior studies examining the response rates of electronic surveys and thus had limited existing research to serve as a benchmark (Mehta \& Sivadas, 1995; Hutchison, et al, 1998). Second, since we randomly selected recipients for the four different conditions, we felt there would be no difference in the likelihood of responses due to the respondents' attitudes toward completing and returning surveys. However, because CPAs use computer technology on a daily basis in the workplace, the researchers believed that the experimental conditions with more technologically oriented response modes would have a higher response rate. Additionally, between the email and WWW response modes, the expectation was that the email response mode would be the highest. 


\section{Research Methodology}

Surveys were distributed via different media to a sample of practicing CPAs listed in the "List of CPA Firms" component of the http://www.cpafirms.com web site. The subjects were randomly assigned to one of four distribution/response conditions with 200 subjects in each condition.

The four distribution/response conditions were developed using combinations of two distribution media and three response media. The distribution media were regular mail and email. The response media were regular mail, email, and web form. The resulting conditions were-specified as distribution/response-regular mail/regular mail (RM/RM), regular mail/regular mail or WWW (RM/RMoWWW), email/email (EM/EM), and email/WWW $(\mathrm{EM} / \mathrm{WWW})$.

Two hundred surveys were distributed for each of the four conditions. In the first condition (RM/RM), efforts were made to simplify returning the surveys. Specifically, an introductory letter, the survey and the return address were all included on one sheet of paper. Upon opening the stapled packet, respondents viewed the brief introductory letter. The letter indicated that participating in the survey consisted of filling in responses, stapling it closed and dropping it in the mail. The survey was on the reverse side of the letter. Postage was prepaid so participants did not incur any expense if they chose to respond.

The surveys for the second condition (RM/RMoWWW) were also sent via regular mail. However, recipients chose to either respond via regular mail or via the WWW using a web site of one of the authors. The URL (Uniform Resource Locator ${ }^{2}$ ) of this web site was included in the letter describing the survey. The survey web form was easy to complete and send back to the researchers. Drop-down option boxes facilitated responses to the questions using a Likert scale.

In the third condition (EM/WWW), potential respondents were sent an email message with the subject title "Accounting Forecasting Exercise." An electronic version of the survey was attached to the email message. This message asked the recipients to respond to the survey by clicking on a "hotlink" included in the message. The "hotlink" connected the recipient to the aforementioned web site where the survey was filled out. The URL was provided in the message if the respondent's email software did not have the "hotlink" option. The respondent then had to personally access the web site by inputting the URL.

The surveys in the fourth condition (EM/EM) were sent via email. A short introductory note indicated that the survey could be answered by using the "Reply" feature included in most email software. Recipients responded by typing their answers to the questions (1-7 on a Likert Scale) in blanks provided for answers.

Both the WWW and email versions of the response methods were tested to ensure functionality. The survey instruments were identical for each of the treatment conditions.

\section{Results}

The actual, overall response rate for this survey was 14 percent, which fell within the expected interval of 5 to 30 percent. The response rates for the four different conditions are shown in Table 1.

Table 1

Response Rates by Distribution and Response Mode

\begin{tabular}{|c|c|c|}
\hline Distribution Mode & Response Mode & Response Rate \\
\hline Regular Mail & Regular Mail & $23 \%$ \\
\hline Regular Mail & World Wide Web & via WWW-0.0\% \\
& Or Regular Mail & $10.5 \%$ \\
\hline Email & World Wide Web & $12 \%$ \\
\hline Email & Email & ial \\
\hline
\end{tabular}


The most obvious difference is among the RM/RM condition and the other three conditions. When survey distribution and response were via regular mail, the response rate was almost twice as high as the other three conditions. This occurred even though there was a small potential bias toward email distribution ${ }^{3}$. Another way to view the data is to collapse the response mode dimension and look at the results based solely on distribution mode. Table 2 shows the data using this view. This difference between distribution modes was tested using a t-test for proportions. The difference was significant $(\mathrm{t}=2.15)$ at the .05 level.

Table 2

Response Rates by Distribution Mode

\begin{tabular}{|c|c|}
\hline Distribution Mode & Response Rate \\
\hline Regular Mail & $16.5 \%$ \\
\hline Email & $11.25 \%$ \\
\hline
\end{tabular}

Additionally, the respondents in condition 2 clearly preferred responding via regular mail as opposed to the WWW. This difference, when tested using a $t$-statistic, was found to be highly significant $(t=6.67)$. A comparison was also made between the two response modes when surveys were distributed via email. This difference was not statistically significant $(\mathrm{t}=-.67)$.

\section{Discussion}

As presented previously, the expectation that more technologically oriented response modes would have a higher response rate was not supported by a statistically significant margin. Also, even though the email response mode resulted in more responses than the WWW response mode, the difference was not significant. Finally, when given a choice of responding using the WWW or regular mail (condition 2), regular mail was chosen unanimously.

The results of this experiment suggest that the choice of distribution mode and response mode in survey research has a significant effect on response rates. There are many possible reasons for this effect. One possible explanation for this difference relates to the capability and knowledge of the response group with respect to regular mail and email. While we assumed all respondents were able to receive regular mail, it is possible some lacked either the capability or the knowledge or both to receive email. The capability to receive email means having the technological infrastructure (e.g. computer, mail server, phone lines, software, etc.) to receive electronic messages. Since all messages were delivered, it seems highly unlikely that any of the firms to which messages were sent lacked capability to receive email. However, the individual CPAs may have lacked the necessary hardware (either by choice or resource constraint) to access email. A more likely scenario is that while the CPAs did have the capability, they did not have the knowledge. This is more likely because email is a recent innovation in communication media.

A second possible reason for the observed difference in response rates is that the respondents were hesitant to read unfamiliar email because of the risk of introducing some type of virus into their system. Computer viruses are often spread via email and one way to reduce the risk of infection is to delete email messages from unfamiliar sources without reading them. Similarly, the survey may have been deleted without being read because the desired respondent may have perceived the survey as "junk email." Because email is cost effective and its usage has significantly increased, it is no surprise that it is being utilized as a medium for advertising and widespread mailings. Regular junk mail is discarded without being opened and the same fate is likely for email messages perceived as "junk email."

The difference in response rates may be explained by the fact that regular mail is tangible and email is intangible. When a survey is received via regular mail, it occupies space. Its presence serves as a passive reminder that it has not been completed. On the other hand, a survey received via email does not occupy physical space in the respondent's work environment. Unless the respondent is viewing email messages received, the email message will not serve as a reminder to respond. This feature of regular mail may have increased the likelihood of responding to the surveys sent via that medium. 
Response rates may have differed because responding to surveys sent via email required more effort than those sent via regular mail. In preparing the surveys for all four conditions, we strove to simplify the response procedure as much as possible. Because the survey was short (seven questions), it probably took less than two minutes to complete. Perhaps because of the ease of completing the regular mail survey, replying via the WWW was perceived to be a more time consuming task, and thus fewer people responded. However, the survey sent via email and responded to either via email or the WWW was comparable to the RM/RM survey in ease of response. Once the message was opened, the respondent in the EM/EM condition had to invoke the reply mode of the email software, type in seven responses and then send the reply. This could easily be done in approximately the same amount of time required to complete the regular mail version. The respondent in the EM/WWW condition responded by clicking on a "hotlink" in the email message. Doing this connected the respondent to the web page that contained the survey. Responding to the survey required the respondent to fill in the date and select a number from 1 to 7 ( 7 point $\mathrm{Li}$ kert scale) from a drop-down option box. The time required to complete this is comparable to the RM/RM and EM/EM conditions unless opening the browser took a relatively longer time (e.g. two or three minutes). In spite of the similarity of ease of completion for the RM/RM, EM/EM and EM/WWW conditions, there was still a significant difference in response rates.

\section{Conclusion}

Researchers employing surveys to gather data have new tools at their disposal with the advent of email and the Internet. The results of this experiment suggest that survey distribution and response modes are important choices to consider in the research planning process. The benefits of reduced costs and greater speed in receiving responses provided by electronic survey options are timely for researchers facing increasingly tight constraints on resources. On the other hand, the restrictive characteristics of electronic data gathering methods must be carefully considered. One critical factor is consideration of the population to whom surveys will be sent. Those possessing the capability and knowledge to reply to surveys via electronic means represent a specific population (e.g. higher socio-economic status and higher education level). If the desired population does not fit this description, choosing an electronic means of gathering data would not be wise. Additionally, even when this specific population is desired, the response rates may not be as high as with a traditional mail survey.

Another conclusion relates to CPAs in the US. An interesting paradox is suggested by the response rate data. If CPAs were equally likely to respond to the survey regardless of distribution and response mode, there must be another explanation for the lower response rate for electronically distributed surveys. One possible explanation is the respondents' treatment of unfamiliar email. Email surveys may have been disregarded because of concerns about computer viruses. The surveys may also have been perceived as "junk email" and were deleted without being opened. Another possible explanation is lack of individual capability and/or knowledge regarding electronic communication. This explanation is consistent with the phenomenon of an inherent hesitancy or resistance to adopt new technology. There is no reason to believe that CPAs would be immune to this phenomenon. If the average age of CPAs responding to the survey was higher than the overall average age of CPAs, this would bolster the idea that some CPAs lack capability and knowledge regarding electronic communication.

\section{Future Research}

The results of this experiment suggest some additional issues to be examined. First, the results suggest the possibility that computer fluency among US CPAs may be lower than expected. Gathering data on computer usage and experience (which could be used as a measure of age) and stratifying by experience/age may provide useful insight about hesitancy on the part of more experienced (older) CPAs to integrate technology in to their work routines. Second, similar data could be gathered from a different group of respondents and then compared to response rates for CPAs. Finally, the effect of technology on other aspects of the workplace, besides communication, could be explored. 


\section{Endnotes}

1. This is true for most software available or this function, although much more expensive and sophisticated software is also available.

2. The Uniform Resource Locater is a unique address for the website.

3. Any email surveys returned as undeliverable were assigned a new address and resent. This process was continued until 200 in each email distribution mode group were delivered. A total of six surveys sent via regular mail were returned as undeliverable. No replacements were sent to new addresses for regular mail surveys.

\section{References}

1. Alreck, Pamela, and Robert B. Settle (1985). The Survey Research Handbook. Homewood, IL: Richard D. Irwin, Inc.

2. Blattberg, Robert C. and Rashi Glazer (1993). "Marketing in the Information Revolution". in Robert C. Blattberg, Rashi Glazer and John D.C. Little (Eds), The Marketing Information Revolution. Boston: Harvard University Press.

3. Dyson, Esther (1993). "If Nothing on E-mail is Private, Just Say So". Computerworld, 27, February 22, p 33.

4. Ferrera, Raymond and Richard L. Nolan (1974), "New Look at Computer Data Entry" in William C. House (ed.) Data Base Management. New York: Petrocelli Books.

5. Hutchinson, Paul D., Gary M. Gleischman and Dean W. Johnson (1998), "E-mail Versus Mail Surveys: A Comparative Study", The Review of Accounting Information Systems, Vol. 2, \#3, pp.43-55.

6. Kiesler, Sara and Lee S. Sproull (1986) "Response Effects in the Electronic Survey". Public Opinion Quarterly, 50, pp 420-13.

7. Odom, Marcus D., Marc A. Giullian and Michael W. Totaro (1999), "New Technology in Survey Research: Does it Improve Response Rates?”, The Review of Accounting Information Systems, Vol. 3, \#1, pp.27-34.

8. Mehta, Raj and Eugene Sivadas, (1995) "Comparing Response Rates and Response Content in Mail Versus Electronic Mail Surveys", Journal of the Marketing Research Society, Vol. 37, \#4, pp 429-39. 